\title{
BMJ Open Establishing a sentinel surveillance system for the novel COVID-19 in a resource-limited country: methods, system attributes and early findings
}

Pritimoy Das (D) , ${ }^{1}$ Zubair Akhtar $10,{ }^{1}$ Syeda Mah-E-Muneer, ${ }^{1}$ Md Ariful Islam, ${ }^{1}$
Mohammed Ziaur Rahman, ${ }^{1}$ Mustafizur Rahman, ${ }^{1}$ Mahmudur Rahman, ${ }^{1}$
Mahbubur Rahman, ${ }^{2}$ Mallick Masum Billah, ${ }^{2}$ ASM Alamgir, ${ }^{2}$
Meerjady Sabrina Flora, ${ }^{2}$ Tahmina Shirin, ${ }^{2}$ Sayera Banu, ${ }^{1}$ Fahmida Chowdhury ${ }^{1}$

To cite: Das P, Akhtar Z, MahE-Muneer S, et al. Establishing a sentinel surveillance system for the novel COVID-19 in a resource-limited country: methods, system attributes and early findings. BMJ Open 2021;11:e055169. doi:10.1136/ bmjopen-2021-055169

- Prepublication history and additional supplemental material for this paper are available online. To view these files, please visit the journal online (http://dx.doi.org/10.1136/ bmjopen-2021-055169).

Received 04 July 2021

Accepted 08 November 2021

Check for updates

(C) Author(s) (or their employer(s)) 2021. Re-use permitted under CC BY-NC. No commercial re-use. See rights and permissions. Published by BMJ.

${ }^{1}$ Infectious Diseases Division (IDD), International Centre for Diarrhoeal Disease Research, Dhaka, Bangladesh

${ }^{2}$ Institute of Epidemiology Disease Control and Research, Dhaka, Bangladesh

Correspondence to Dr Pritimoy Das; pritimoydas@gmail.com

\section{ABSTRACT}

Objectives To establish a hospital-based platform to explore the epidemiological and clinical characteristics of patients screened for COVID-19.

Design Hospital-based surveillance.

Setting This study was conducted in four selected hospitals in Bangladesh during 10 June-31 August 2020.

Participants In total, 2345 patients of all age (68\% male) attending the outpatient and inpatient departments of surveillance hospitals with any one or more of the following symptoms within last 7 days: fever, cough, sore throat and respiratory distress.

Outcome measures The outcome measures were COVID-19 positivity and mortality rate among enrolled patients. Pearson's $\chi^{2}$ test was used to compare the categorical variables (sign/symptoms, comorbidities, admission status and COVID-19 test results). Regression analysis was performed to determine the association between potential risk factors and death.

Results COVID-19 was detected among 922 (39\%) enrolled patients. It was more common in outpatients with a peak positivity in second week of July $(112,54 \%)$. The median age of the confirmed COVID-19 cases was 38 years (IQR: $30-50), 654$ (71\%) were male and $83(9 \%)$ were healthcare workers. Cough $(615,67 \%)$ was the most common symptom, followed by fever $(493,53 \%)$. Patients with diabetes were more likely to get COVID-19 than patients without diabetes (48\% vs $38 \%$; OR: $1.5 ; 95 \% \mathrm{Cl}$ : 1.2 to 1.9). The death rate among COVID-19 positive was $2.3 \%, n=21$. Death was associated with age $\geq 60$ years (adjusted OR (AOR): 13.9; 95\% Cl: 5.5 to 34), shortness of breath (AOR: $9.7 ; 95 \% \mathrm{Cl}: 3.0$ to 30 ), comorbidity (AOR: 4.8; $95 \% \mathrm{Cl}: 1.1$ to 21.7 ), smoking history (AOR: 2.2, $95 \% \mathrm{Cl}: 0.7$ to 7.1 ), attending the hospital in $<2$ days of symptom onset due to critical illness (AOR: $4.7 ; 95 \% \mathrm{Cl}$ : 1.2 to 17.8 ) and hospital admission (AOR: $3.4 ; 95 \% \mathrm{Cl}: 1.2$ to 9.8$)$.

Conclusions COVID-19 positivity was observed in more than one-third of patients with suspected COVID-19 attending selected hospitals. While managing such patients, the risk factors identified for higher death rates should be considered.
Strengths and limitations of this study

- The sentinel surveillance for COVID-19 was implemented in collaboration with a government public health institution and an international research organisation in resource-constrained settings.

- This was a multicentre study with representative hospitals included from almost all major administrative regions of Bangladesh.

- Our surveillance method was unique as we shared test results with patients at earliest and considered follow-up at 30-day after enrolment to track the prognosis of COVID-19-positive patients even after they were discharged from the enrolling hospital.

- As we did not test any asymptomatic patients and community burden estimation was beyond our scope, the true prevalence of patients with COVID-19 might be higher than reported in our study.

\section{INTRODUCTION}

Starting from its inception at Wuhan, Hubei Province, China, the novel coronavirus named SARS-CoV-2 has spread across the world within a few months, causing COVID-19. ${ }^{1}$ Globally, 129651305 cases and 2.8 million deaths were recorded until 31 March 2021. ${ }^{2}$ This virus manifests various clinical characteristics, from asymptomatic infection to severe pneumonia, vasculitis and death. ${ }^{3-6}$ It was declared a public health emergency of international concern by the WHO on 30 January 2020 and subsequently a pandemic on 11 March $2020 .^{7}$ During that early stage of this coronavirus disease, there was uncertainty and variation regarding the epidemiological, clinical, and virological characteristics of this novel infectious disease. Though COVID-19 cases were reported from 198 countries or regions, and over 400000 people were confirmed to be infected globally 
(24 March 2020), ${ }^{8}$ its transmission dynamics within the human population were unclear, so the WHO designed a protocol for the countries to investigate the COVID-19 outbreaks locally and emphasised COVID-19 surveillance to understand the country situation. ${ }^{9}$

Bangladesh, a country in Southeast Asia, exhibited different epidemiological features compared with other countries regarding the influenza virus in terms of seasonality, severity and mortality. ${ }^{11}$ On 8 March 2020, the first three cases of confirmed COVID-19 were reported in Bangladesh, ${ }^{12}$ and subsequently, the number of confirmed cases and deaths increased: at the end of the first month, there were 51 confirmed cases with 5 deaths from COVID-19. ${ }^{13}$ As COVID-19 was a novel virus, there was minimal information regarding its severity and magnitude in Bangladesh.

The government of Bangladesh ( $\mathrm{GoB})$ initiated several efforts for the early detection of the virus to mitigate the spread such as screening of passengers at airports, land ports and maritime ports; hotline system to notify any suspected case of COVID-19 to the Institute of Epidemiology, Disease Control and Research (IEDCR) so that their specimens could be collected and tested. Moreover, passengers arriving from countries with COVID-19 outbreaks were screened at the point of entries and monitored for any symptom onset for 14 days, considering the virus's incubation period recommended by the WHO. ${ }^{14}$ However, these efforts were not enough to detect patients with COVID-19, as asymptomatic COVID-19 carriers already unfolded in their community and spread the virus in different geographical locations across Bangladesh. ${ }^{15}$ Patients with COVID-19 symptoms were reported from different hospitals and needed to be tested for diagnosis and appropriate treatment purposes. ${ }^{16}$ Initially, there were 10 laboratories in capital Dhaka city and 5 laboratories outside Dhaka had COVID-19 testing facility in Bangladesh. ${ }^{17}$ Thus, as a part of the pandemic preparedness and responses, there was an immediate need to establish a hospital-based platform to screen patients with suspected COVID-19 to support GoB in hospitals where PCR-based COVID-19 testing facility was not available. The GoB initiated a countrywide system for detecting COVID-19 cases by prioritising divisional hospitals, medical college hospitals, and few specialised hospitals to screen and test for COVID-19. Moreover, there was a knowledge gap on clinical and epidemiological data of patients with COVID-19 in Bangladesh during the first wave of the pandemic from any sentinel sites involving multiple public and private hospitals across the country.

The quality of surveillance data in many developing countries is hampered by a variety of factors, including a lack of resources and training. ${ }^{18}$ Ibrahim looked into various COVID-19 surveillance activities around the world and categorised them into a systematic review paper of 30 articles. ${ }^{19}$ Our surveillance falls into a combination of sentinel surveillance and enhanced surveillance of hospitalised cases in which risk groups can be identified, tested, and followed up on via a hospital and laboratory network.
Current surveillance included searching for patients with suspected COVID-19 among hospitalised patients as well as screening and testing patients from outpatient. In Singapore, a similar approach was taken for the investigation and confinement efforts for COVID-19. ${ }^{20} 21$

To support the containment efforts for COVID-19, the International Centre for Diarrhoeal Disease Research, Bangladesh (icddr,b) and the IEDCR under the Bangladesh Ministry of Health and Family Welfare jointly conducted this surveillance in selected hospitals where there was no nearby PCR-based COVID-19 testing facility. The aim of this study was to establish a hospital-based platform to describe and analyse epidemiological and clinical characteristics of patients screened for COVID-19 in selected Bangladeshi hospitals with limited resources during the first wave of the COVID-19 pandemic.

\section{METHODS}

\section{Setting}

The surveillance was conducted at the outpatient department (OPD) and inpatient department (IPD) of four selected hospitals where patients sought healthcare with suspected COVID-19 symptoms. There were three public hospitals and one private hospital, all of which were in different geographical locations across Bangladesh (figure 1). The public hospitals namely Sadar Hospital, Habiganj (24²2'24.77”, 91 $\left.{ }^{\circ} 25^{\prime} 3.62^{\prime \prime}\right)$, General Hospital, Patuakhali $\left(22^{\circ} 21^{\prime} 52.19^{\prime \prime}, 90^{\circ} 19^{\prime} 37.25^{\prime \prime}\right.$ and District Hospital, Narshingdi (235' $\left.48.6^{\prime \prime}, 90^{\circ} 42^{\prime} 9.84^{\prime \prime}\right)$, all having 100-250 numbers of beds. Jahurul Islam Medical College Hospital, Kishoregonj (24¹2'2.26”, 9055’1.81”) is a general tertiary-level 500-bed teaching hospital. To select these hospitals, we evaluated the ongoing national hospital-based influenza surveillance platforms to identify the hospitals where there was no in-hospital or nearby PCR-based COVID-19 testing facility at that time but a high load of potential patients with suspected COVID-19 in that geographical location. It was considered that additional support to these hospitals would strengthen COVID-19 case identification and reporting at the national level with generation of epidemiological data.

\section{Patient enrolment}

Within 3 months of the first COVID-19 case detection in the country, we deployed two trained field staff in each selected hospital (total eight field staff placed in four hospitals) for screening patients with suspected COVID-19 among all the patients attending the fever clinic at OPD and among all inpatients admitted into the specific wards (medicine ward, paediatric ward, intensive care unit (ICU) and COVID-19 isolation ward). These field staff worked with hospital physicians to enrol suspected patients with COVID-19.

Case definition

Field staff actively screened for patients with suspected COVID-19 using the following case definition: patient 


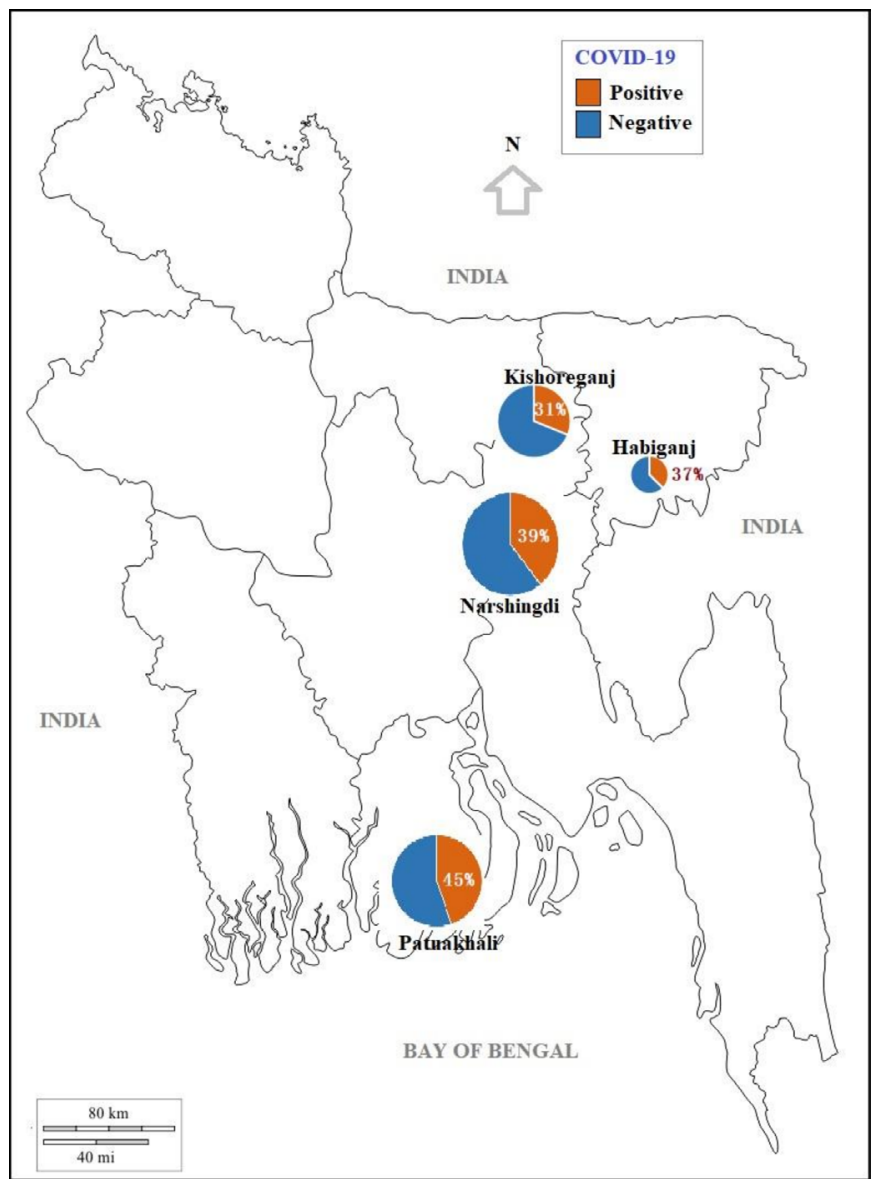

Figure 1 Location of the study hospitals and proportionate distribution of enrolled patients at different sites with their COVID-19 positivity, June to August 2020

with any one or more of the following symptoms within last 7 days-fever, cough, sore throat and respiratory distress. This case definition was applied by GoB to collect samples as patients with suspected COVID-19.

\section{Data collection}

After obtaining written informed consent from patients who met the suspected COVID-19 case definition, field staff collected data on sociodemographics (age, sex, occupation, educational level), travel history (local or international travel) and clinical characteristics (presenting symptoms, clinical signs, comorbidity, admission status, smoking history, duration of symptom onset to treatment seeking) from them. Field staff used proper personal protective equipment (PPE) such as N95 mask/medical mask, disposable gown, disposable cap, disposable gloves, face shield and goggles during data and specimen collection. Field staff used tablet computers to collect data syncing with local icddr,b server using mobile internet. This system allowed real-time monitoring of the situation across all hospitals by the research team centrally from Dhaka city. After 30 days of enrolment, the surveillance team (field staff, their supervisor and occasionally, the first author) followed up with each enrolled patient through mobile phone calls to register the outcome of their illnesses and updated the database accordingly. The outcome variables were COVID-19 positivity by reverse transcription (RT)-PCR test and the mortality among the SARS-CoV-2-infected patients.

\section{Specimen collection and transportation}

Trained field staff collected a single nasopharyngeal swab through swab stick from each enrolled patients in viral transportation medium (VTM) and stored in a cool box between $2^{\circ} \mathrm{C}$ and $4^{\circ} \mathrm{C}$ temperature. In-house (icddr,b lab) VTM preparation was used for the collected samples. Every afternoon, a dedicated porter transported all the samples to icddr,b, Dhaka using a private car from three surveillance hospitals except Patuakhali. From Patuakhali, one of the dedicated porter brought samples to icddr,b by launch (public transport). All VTMs were handed over to icddr,b virology laboratory within 24 hours of specimen collection.

\section{Laboratory testing}

Nasopharyngeal swabs were tested for SARS-CoV-2 at the virology laboratory of icddr,b. RNA was extracted from nasopharyngeal swab using QiaAmp Viral RNA Mini kit (Qiagen, Hilden, Germany). RNA was tested for SARS-CoV-2 by real-time RT-PCR reaction (rRTPCR) targeting SARS-CoV-2-specific ORFlab-gene and $\mathrm{N}$-gene. Any person with an rRT-PCR positive test result was defined as a case/patient with laboratory-confirmed COVID-19.

\section{Reporting to IEDCR, surveillance hospitals and patients}

We received the laboratory test results on the following day of specimen collection. Our research team then shared the results with respective hospital authorities, district civil surgeons, divisional health directors and the director of IEDCR over email. Moreover, we sent a text message (Short Message Service) to each enrolled patient informing their test report within 36 hours of specimen collection. Our investigators also responded to every query when any COVID-19-positive patient called them over the telephone upon getting the test result. The respective healthcare facilities then managed the patients following the existing government system.

\section{Data analysis}

The data management and analysis were performed using the software Stata V.13 (Stata Corp, College Station, Texas, USA). We summarised all categorical variables using frequency and percentage. We also summarised using mean and SD for symmetrically distributed variables and median and IQR for asymmetrically distributed variables. We performed Pearson's $\chi^{2}$ test to compare the categorical variables and considered $\mathrm{p}<0.05$ as statistically significant. We used univariate logistic regression analysis for strengths of associations and identified risk factors for death, using OR and adjusted for age and sex in the multivariable model. 


\section{Patient and public involvement}

Patients or the public were not involved in the study design, or conduct, or reporting, or dissemination plans.

\section{RESULTS}

COVID-19 positivity and demographic characteristics

During 10 June-31 August 2020, we enrolled 2345 patients with suspected COVID-19 from four selected hospitals. Virology laboratory of icddr,b tested all the 2345 nasopharyngeal swab samples collected from these enrolled participants; of them, 922 (39.3\%) were patients with laboratory-confirmed COVID-19. The median age of the patients with confirmed COVID-19 was 38 years (IQR:
30-50 years), and $654(71 \%)$ were male. COVID-19 was mostly detected among patients aged between 21 and 40 years $(474,51.3 \%)$. About half of the COVID-19-positive patients $(467,50.7 \%)$ had a higher level of education (>12 years). We identified 302 (13\%) of the patients meeting surveillance case definition were healthcare workers (HCWs), and they constituted $83(9 \%)$ of all patients with confirmed COVID-19 (table 1).

\section{Seasonality and geographical variation}

Over the 3 months of the surveillance period, the peak of the COVID-19 positivity among patients with suspected COVID-19 was detected in the 24th and 25th epi weeks (second and third week of July 2020). We observed

Table 1 Sociodemographic characteristics of patients with suspected COVID-19 in selected hospitals of Bangladesh, JuneAugust 2020

\begin{tabular}{|c|c|c|c|c|}
\hline \multirow[b]{2}{*}{ Characteristics } & \multirow{2}{*}{$\begin{array}{l}\text { Patients with } \\
\text { suspected COVID-19 } \\
\text { ( } \mathrm{N}=2345)\end{array}$} & \multicolumn{3}{|c|}{ SARS-CoV-2 positive by rRT-PCR } \\
\hline & & Total positive (922) & $\begin{array}{l}\text { Inpatient (57) } \\
\mathrm{n}(\%)\end{array}$ & $\begin{array}{l}\text { Outpatient (865) } \\
\text { n (\%) }\end{array}$ \\
\hline \multicolumn{5}{|l|}{ Age (in years) } \\
\hline Median (IQR) & $35(26-48)$ & $38(30-50)$ & $55(45-69)$ & $38(29-48)$ \\
\hline \multicolumn{5}{|l|}{ Age subgroups } \\
\hline $0-5$ & $25(1.11)$ & $6(0.7)$ & $1(1.7)$ & $5(0.6)$ \\
\hline $6-10$ & $30(1.3)$ & $11(1.2)$ & $0(0)$ & $11(1.3)$ \\
\hline $11-20$ & $167(7.1)$ & $42(4.5)$ & $0(0)$ & $42(4.8)$ \\
\hline $21-30$ & $684(29.1)$ & $228(24.7)$ & $5(8.7)$ & $223(25.8)$ \\
\hline $31-40$ & $594(25.3)$ & $246(26.6)$ & $6(10.5)$ & $240(27.7)$ \\
\hline $41-50$ & $382(16.3)$ & $182(19.7)$ & $11(19.3)$ & $171(19.7)$ \\
\hline $51-60$ & $267(11.4)$ & $125(13.5)$ & $8(14.0)$ & $117(13.5)$ \\
\hline $60+$ & $196(8.3)$ & $82(8.8)$ & $26(45.6)$ & $56(6.5)$ \\
\hline \multicolumn{5}{|l|}{ Sex } \\
\hline Male & $1590(67.8)$ & $654(70.9)$ & $38(66.7)$ & $616(71.2)$ \\
\hline Female & 755 (32.2) & $268(29.1)$ & 19 (33.3) & $249(28.8)$ \\
\hline \multicolumn{5}{|l|}{ Occupation } \\
\hline $\mathrm{HCW}$ & 302 (12.9) & $83(9.0)$ & $2(3.5)$ & $81(9.4)$ \\
\hline Service & $946(40.3)$ & $431(46.8)$ & $7(12.3)$ & $424(49.0)$ \\
\hline Business & $154(6.6)$ & $82(8.9)$ & $6(10.5)$ & $76(8.8)$ \\
\hline Student & $223(9.5)$ & $68(7.4)$ & $0(0)$ & $68(7.9)$ \\
\hline Dependent & $215(9.1)$ & $73(7.9)$ & $22(38.6)$ & $51(5.9)$ \\
\hline Unemployed & $145(6.1)$ & $64(6.9)$ & $9(15.8)$ & $55(6.3)$ \\
\hline Others* & $360(15.3)$ & $121(13.1)$ & $11(19.3)$ & $110(12.7)$ \\
\hline \multicolumn{5}{|l|}{ Education (years) } \\
\hline No formal schooling & $155(6.6)$ & $44(4.8)$ & $11(19.3)$ & $33(3.8)$ \\
\hline $1-5$ & 255 (10.9) & $89(9.7)$ & $11(19.3)$ & $78(9.0)$ \\
\hline $6-10$ & $496(21.2)$ & 178 (19.3) & 22 (38.6) & $156(18.0)$ \\
\hline $11-12$ & 367 (15.7) & $144(15.6)$ & $7(12.3)$ & $137(15.8)$ \\
\hline$>12$ & $1072(45.6)$ & $467(50.7)$ & $6(10.5)$ & $461(53.4)$ \\
\hline
\end{tabular}

${ }^{*}$ Farmer, day labour, small shop owner, rickshaw/van puller, driver, etc.

HCW, healthcare worker; rRT-PCR, real-time reverse transcription-PCR. 

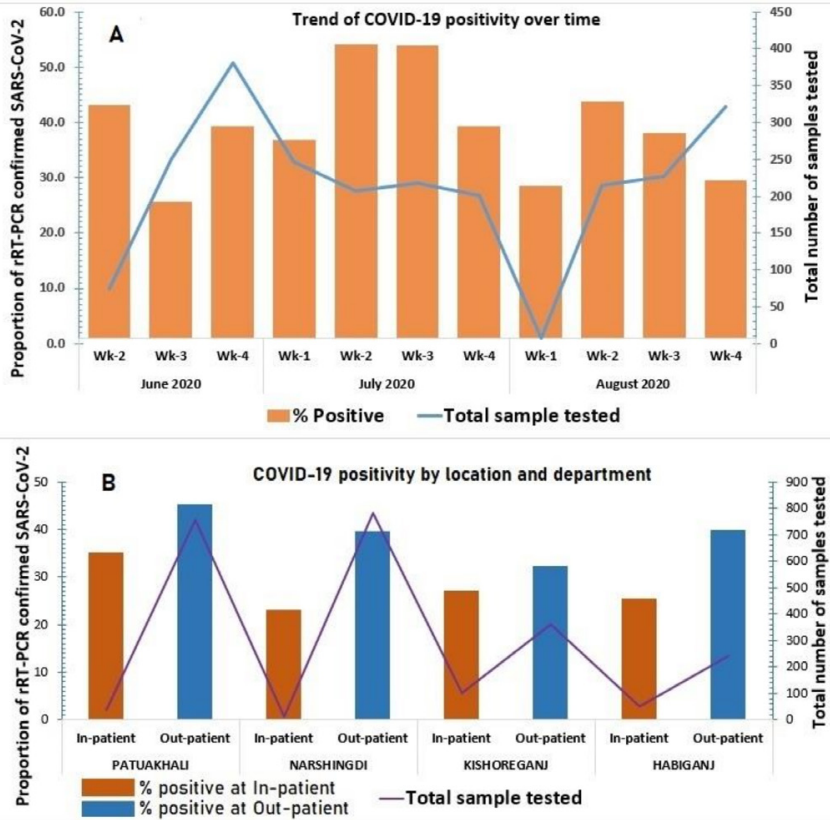

Figure 2 SARS-CoV-2 infection among suspected COVID-19 patients at inpatient and outpatient departments of selected hospitals during June to August 2020, Bangladesh. A. Detection of SARS-CoV-2 at all four selected hospitals over time. B. Detection of SARS-CoV-2 at inpatient and outpatient departments of selected hospital sites. rRT-PCR, real-time reverse transcription-PCR.

a relatively hard-to-reach riverside area (Patuakhali Hospital) reporting the highest number and proportion of cases (355 of 793; 45\%) compared with other hospitals in Narshingdi (313 of 796; 39\%), Kishoreganj (144 of 462; $31 \%$ ) and Habiganj (110 of 294; 37\%). The proportion of test positivity over time dropped and gradually started declining from the epi week 28th (second week of August) (figures 1 and 2A,B).

\section{Differences in clinical presentation between COVID-19- positive and COVID-19-negative patients}

The presenting clinical features in all patients with suspected COVID-19 varied from dry cough (most common, $67 \%, \mathrm{n}=1562$ ) to rash (least common, $0.4 \%$, $\mathrm{n}=9$ ) (figure $3 \mathrm{~A})$. We found fever $\geq 38^{\circ} \mathrm{C}(1252,53 \%$ vs $1077,44 \%)$, loss of taste $(964,41 \%$ vs $711,30 \%)$, headache $(772,33 \%$ vs $645,27 \%)$, fatigue $(696,30 \%$ vs 499 , $21 \%)$, loss of smell $(528,23 \%$ vs $295,13 \%)$, nausea/ vomiting $(431,18 \%$ vs $340,15 \%)$ and joint pain $(314,13 \%$ vs $223,9 \%$ ) were more likely to be the presenting clinical features among COVID-19-positive patients compared with the negative patients $($ all $\mathrm{p}<0.05)$. On the other hand, though sore throat was a very common feature among patients with suspected COVID-19, it was less common in the COVID-19-positive patients than COVID-19-negative patients $(900,38 \%$ vs $1060,45 \%, p=0.002)$ (figure $3 \mathrm{~A}$ ).

\section{IPD versus $O P D$ visits}

Most of the patients meeting the suspected COVID-19 case definition $(2141,91 \%)$ were identified from the

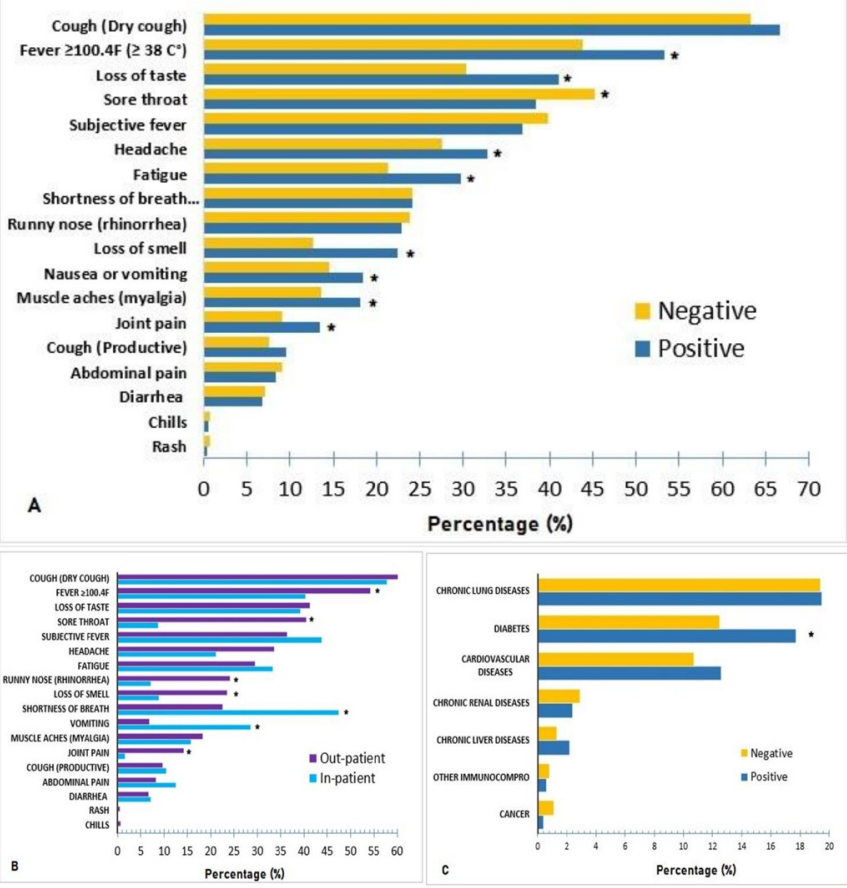

Figure 3 .Distribution of SARS-CoV-2 infected patients by their clinical features and comorbidity in selected hospitals of Bangladesh, June-August 2020. A. Presenting symptoms of all COVID-19 suspected patients by rRT-PCR results. B. Presenting symptoms of COVID-19 patients by department. C. COVID-19 positive patients by their comorbidity.

OPDs of all the surveillance hospitals; and of them, 865 $(40 \%)$ were COVID-19 positive. In contrast, among all patients enrolled from the IPDs, 57 (28\%) were found COVID-19 positive. Shortness of breath (97, $47 \%$ vs 482 , $22 \%)$ and vomiting $(58,29 \%$ vs $146,7 \%)$ were more common clinical characteristics of COVID-19-positive patients admitted in the hospitals compared with patients with COVID-19 who attended at outpatients (figure 3B). However, fever $(1163,54 \%$ vs $82,40 \%)$, sore throat (869, $41 \%$ vs $18,9 \%)$, runny nose $(518,24 \%$ vs $14,7 \%)$, loss of smell $(503,24 \%$ vs $18,9 \%)$ and joint pain $(304,14 \%$ vs $3,2 \%$ ) were more common clinical symptoms in patients with COVID-19 at OPD compared with patients with COVID-19 at IPD (all p<0.05).

\section{Comorbidities among patients with COVID-19}

Compared with COVID-19-negative patients, patients with comorbidities reported more infection with SARSCoV-2, such as for chronic liver disease $(20,51 \%$ vs 898 , $39 \%)$, cardiovascular disease $(116,43 \%$ vs $801,39 \%)$ and diabetes $(164,48 \%$ vs $755,38 \%)$. Of these comorbidities, patients with diabetes showed significantly high susceptibility $(\mathrm{p}<0.05)$ of getting infected with SARS-CoV-2 than patients without diabetes (figure 3C). Other than these comorbidities, we also found 19 patients with cancer meeting enrolment criteria; of them, four patients (21\%) were COVID-19 positive; and among positive, one (25\%) died. 
Table 2 Factors associated with adverse outcome (death) among COVID-19-positive patients in selected hospitals of Bangladesh, June-August 2020

\begin{tabular}{|c|c|c|c|c|c|}
\hline Factors & & $\begin{array}{l}\text { Frequency } \\
\mathrm{N}=922\end{array}$ & $\begin{array}{l}\text { Death } n=21 \\
\text { Frequency (\%) }\end{array}$ & $\begin{array}{l}\text { OR } \\
(95 \% \mathrm{Cl})\end{array}$ & $\begin{array}{l}\text { Adjusted OR } \\
(95 \% \mathrm{Cl})\end{array}$ \\
\hline \multirow[t]{2}{*}{$\mathrm{Age}^{\star}$} & $0-59$ years & 812 & $8(1)$ & 1 & 1 \\
\hline & $\geq 60$ years & 110 & $13(11.8)$ & 13.5 (5.4 to 33.3$)$ & 13.9 (5.5 to 34.5$)$ \\
\hline \multirow[t]{2}{*}{ Sex } & Male & 654 & $14(2.1)$ & 1 & 1 \\
\hline & Female & 268 & $7(2.6)$ & 1.2 (0.5 to 3.0$)$ & 1.5 (0.55 to 4.0$)$ \\
\hline \multirow[t]{2}{*}{ Healthcare worker } & Yes & 83 & $0(0)$ & 1 & 1 \\
\hline & No & 839 & $21(2.5)$ & - & - \\
\hline \multirow[t]{2}{*}{ Symptoms } & $\leq 3$ symptoms & 314 & $7(2.2)$ & 1 & 1 \\
\hline & >3 symptoms & 608 & $14(2.3)$ & $1.0(0.4$ to 2.5$)$ & $1.4(0.52$ to 3.9$)$ \\
\hline \multirow[t]{2}{*}{ Shortness of breath* } & No & 700 & $4(0.6)$ & 1 & 1 \\
\hline & Yes & 222 & $17(7.7)$ & 14.4 (4.8 to 43$)$ & 9.7 (3.0 to 30.4$)$ \\
\hline \multirow[t]{2}{*}{ Comorbidity* } & No & 538 & $2(0.4)$ & 1 & 1 \\
\hline & Yes & 384 & $19(4.9)$ & $13.9(3.2$ to 60$)$ & 4.8 (1.05 to 21.7$)$ \\
\hline \multirow[t]{2}{*}{ History of smoking } & No & 812 & $14(1.7)$ & 1 & 1 \\
\hline & Yes & 110 & $7(6.4)$ & 3.9 (1.5 to 9.8$)$ & 2.2 (0.71 to 7.1$)$ \\
\hline \multirow[t]{2}{*}{ Treatment received ${ }^{*}$} & OPD & 865 & $12(1.4)$ & 1 & 1 \\
\hline & Inpatient & 57 & $9(15.8)$ & 13.3 (5.3 to 33$)$ & $3.4(1.2$ to 9.8$)$ \\
\hline \multirow{2}{*}{$\begin{array}{l}\text { Duration of hospital } \\
\text { attendance from the } \\
\text { onset of symptom* }\end{array}$} & $\geq 2$ days & 880 & $17(1.9)$ & 1 & 1 \\
\hline & $<2$ days & 42 & $4(9.8)$ & 5.4 (1.8 to 17.1$)$ & $4.7(1.2$ to 17.8$)$ \\
\hline
\end{tabular}

${ }^{*}$ Factors with a significant difference between groups.

OPD, outpatient department.

\section{Mortality and associated risk factors}

Among the 922 patients with laboratory-confirmed COVID-19, 21 (2.3\%) patients were reported dead from our routine follow-up after a month of enrolment. Of them, $76 \%$ (16 of 21) patients died at the same enrolment hospital or different hospitals, 24\% (5 of 21) patients died at home or on the way to the hospital. From the onset of symptoms, $43 \%$ (9 of 21) of patients died within 7 days, and $95 \%$ (20 of 21) deaths occurred within 15 days (online supplemental table 1). When we compared epidemiological factors for association with adverse outcome of their clinical progression, our data showed that death was more likely to occur among patients presenting with age $\geq 60$ years (adjusted OR (AOR): 13.9; 95\% CI: 5.5 to 34.5 ), shortness of breath (AOR: 9.7; 95\% CI: 3.0 to 30), comorbidity (AOR: 4.8; 95\% CI: 1.1 to 21.7), smoking history (AOR: 2.2, 95\% CI: 0.7 to 7.1), attending to a hospital in $<2$ days from the onset of symptoms due to critical illness (AOR: 4.7; 95\% CI: 1.2 to 17.8 ) and hospital admission (AOR: 3.4 ; $95 \%$ CI: 1.2 to 9.8 ; table 2 ).

\section{DISCUSSION}

Our hospital-based COVID-19 sentinel surveillance platform identified more than one-third (39\%) of patients as laboratory-confirmed COVID-19 among the patients with suspected COVID-19 attending the hospitals during the study period. This COVID-19 positivity rate was much higher to draw public health attention compared with the WHO reported national data $(19.5 \%)$ from 8 March to 14 September $2020 .^{22}$ The national health system intended to collect specimens from symptomatic individuals, but considering resource-constrained verification of symptoms was difficult and thereby some asymptomatic individuals could be included for testing. Moreover, people seeking a routine COVID-19 PCR test as a requirement for international travel was also included in the national system for COVID-19 reporting. In contrast, surveillance staff and physicians strictly verified the symptoms reported by each patient before enrolment and sample collection through the sentinel surveillance platform. This sentinel surveillance was strictly supervised and monitored by a team of dedicated researchers for the utmost quality of specimen and data collection from actual symptomatic patients and rapid transportation of specimens from remote field sites to central laboratory at Dhaka maintaining recommended standard temperature for rRT-PCR testing. Thereby, a robust sentinel COVID-19 surveillance is so important to better understand the actual disease burden in different administrative regions of a country.

More than half of our COVID-19-positive patients were young adults within the age group of 21-40 years. This was consistent $(46.7 \%)$ with the WHO report for 
Bangladesh on morbidity and mortality weekly update as of 14 September 2020. ${ }^{22}$ Among the COVID-19-positive patients, male was predominant. This was consistent with other nearby countries such as India, where researcher reported that male COVID-19 cases $(65.39 \%)$ were more than females $(34.61 \%){ }^{23}$ This might be due to the maledominant societies' unique health-seeking behaviour like Bangladesh, where women do not seek healthcare unless severe. ${ }^{24}$ This finding was similar (68\% male) during March-April, the early phase of novel coronavirus detection in Bangladesh. ${ }^{25}$ Other than male predominance, more than half of the COVID-19-positive patients were found to be educated for 12 years or more. This might not be the cause that educated people were more infected than less educated or uneducated; rather it may be people with higher education were more conscious and thus were coming to hospitals for testing.

Among COVID-19-suspected HCWs from our surveillance hospitals, one out of four was SARS-CoV-2 infected (27\%). Chatterjee et al reported that $5 \%$ of symptomatic HCWs were SARS-CoV-2 positive in India. ${ }^{26}$ This was not a surprise as HCWs remain vulnerable to infectious disease in any low/middle-income countries such as Bangladesh and demand adequate preparedness to fight against COVID-19. ${ }^{27}$ Our findings also support that HCWs were getting infected at a much higher rate than India and, even more than that of a COVID-19-dedicated tertiary care hospital in Dhaka $(11 \%),{ }^{28}$ Bangladesh. Therefore, appropriate measures should be taken to prevent primary infection from patients and secondary infection from colleagues. Appropriate measures such as infection prevention and control training, adequate PPE supply and their proper use should be taken into consideration with high priority to protect HCWs from getting infected from their workplace.

The positivity rate helps public health officials to assess the disease burden at different time points. COVID-19 positivity rate among the tested patients was increasing until July, followed by a gradual decline, similar to the country trend as reported by the Director General of Health Services Bangladesh and WHO report. ${ }^{29} 30$ There was a sharp drop in specimen collection in the first week of August due to 'Eid holidays', the biggest religious festival for Muslims.

During the initial days of the COVID-19 crisis, there was a deficiency of adequate data to make appropriate policy decisions for Bangladesh. ${ }^{31}$ Providing timely test reports and feeding peripheral sites' data from our surveillance hospitals to the government recording system greatly enhanced the management of the novel coronavirus crisis. Moreover, our work generated some key information about the ongoing COVID-19 pandemic in Bangladesh. COVID-19-suspected patients were found more at OPD than IPD, indicating that patients with COVID-19 symptoms were primarily mild, thereby seeking treatment from the OPD. Clinical features widely vary from asymptomatic infection (40\%-45\% of SARS-CoV-2 infections) to death from acute respiratory distress syndrome. ${ }^{56} 32$
Chinese researchers reported fever, cough and expectoration were the most common symptoms ${ }^{33}$ in a multicentred study. Another meta-analysis ${ }^{34}$ revealed fever $(88.7 \%)$, cough $(57.6 \%)$ and dyspnoea $(45.6 \%)$ were the prominent presentation. We found cough followed by fever as the top two presenting symptoms of patients with COVID-19. Additionally, sore throat, loss of taste and loss of smell, headache, and muscle and joint pain were more likely to occur among patients with laboratory-confirmed COVID-19. Nothing conclusive, but these differences could be used carefully by the treating physicians to manage a patient with suspected COVID-19 initially before getting the lab report.

Comorbidities play a crucial role towards disease progression. Diabetes was the most commonly reported factor towards the adverse outcome of patients with COVID-19 and their disease progression, ${ }^{35}$ requiring more hospitalisation in the ICU and associated with more death compared with patients with COVID-19 without diabetes. ${ }^{36}$ Our surveillance data showed that patients with diabetes were more susceptible to get a COVID-19 infection than others. So, besides other comorbidities such as cardiovascular diseases, clinicians should consider additional clinical measures to manage a COVID-19positive patient with diabetes.

Mortality rate is one of the key indicators in public health. Our surveillance data revealed that the percentage of death among our COVID-19-positive patients was a little higher $(2.3 \%)$ than the global average death rate $(2.2 \%)$ as of 22 March 2021. ${ }^{2}$ This death rate was possible to capture due to the unique post-discharge telephone follow-up of enrolled patients after 30 days of enrolment for their outcome. Among all COVID-19-positive patients identified through the surveillance, we detected only $28 \%$ deaths from the surveillance hospitals, the remaining $72 \%$ deaths were possible to capture from this unique follow-up strategy of our sentinel surveillance system.

We observed elderly, comorbidity, having breathing difficulty, smoking and admission in the IPD due to more severe illness were more likely to be the risk factors for death among the SARS-CoV-2-infected patients. Regarding gender, Italy reported more death among men than women. ${ }^{37}$ Nationally, Bangladesh has more COVID-19 deaths among men (76\%) than women $(24 \%),{ }^{38}$ but we did not find any significant difference in death rate between men and women from our surveillance. A nationwide analysis in China showed that age between 65 and 74 years, coronary heart disease, cerebrovascular disease and dyspnoea were independent risk factors associated with fatal outcome. ${ }^{39}$ The Chinese Center for Disease Control and Prevention analysed 44000 data of patients with COVID-19 and reported elderly, diabetes, cardiovascular disease, hypertension and chronic respiratory disease were all associated with an increased risk of death. ${ }^{40}$ In the UK, people aged over 70 years with cardiovascular and respiratory diseases were considered as high-risk group. ${ }^{41}$ Smoking was associated with increased risks of COVID-19 death and disease 
progression, a finding similar to other studies. ${ }^{42-45}$ The WHO also mentioned increased severity of disease and mortality in hospitalised patients with COVID-19 among smokers. ${ }^{46}$ One meta-analysis reported a pooled OR of 1.89 (95\% CI: 1.10 to 3.24 ) on the severity of this disease among smokers than non-smokers. ${ }^{44}$ Another systematic review reported a significant association between smoking and the progression of COVID-19 (OR: 1.91; 95\% CI: 1.42 to 2.59 ); the authors commented that the actual risk of smoking might be higher. ${ }^{47}$ Therefore, for a better outcome from COVID-19 infection during this pandemic, smoking should be avoided. The surveillance data also showed more deaths occurred among critically ill hospitalised patients, which is natural. Therefore, an improved referral system from a district-level hospital to a tertiary-level or specialised hospital could be considered for high-risk patients, which might reduce mortality.

Despite all efforts, our work had certain limitations. Based on our available resources and government priority, we conducted this surveillance at only four hospitals in different locations and enrolled suspected patients for 3 months only, with an additional 1-month follow-up period. Thus, our findings might not be generalised for the whole Bangladeshi population. Moreover, we might have missed the true prevalence of patients with COVID-19 as we did not screen any asymptomatic patients. We only reported hospital-based prevalence because it was beyond the scope of this platform to estimate the community burden of COVID-19 in Bangladesh.

\section{CONCLUSION}

Of the patients attending the surveillance hospitals with COVID-19 symptoms during the reporting period, more than one-third had laboratory-confirmed COVID-19 and, this was more common among outpatients with peak positivity in July. Elderly population, shortness of breath, comorbid condition, smoking history and severe illness requiring hospital treatment were identified as the factors associated with death among patients with COVID-19. Policymakers may consider a system for the early identification of the COVID-19-positive individuals at high risk to provide special care with time-appropriate treatment. Our effort strengthened government's capacity for rapid case detection, reporting and quick containment efforts. Continuing this sentinel surveillance platform can better characterise disease patterns in populations over time, thus support the government by assessing the magnitude of the health problem and developing a data-driven effective management strategy as well as monitor the progress towards the reduction of COVID-19 cases after vaccination campaign for SARS-CoV-2.

Acknowledgements The icddr,b acknowledges with gratitude the commitment of FCDO and USAID to its research efforts. icddr,b is also grateful to the governments of Bangladesh, Canada, Sweden and the UK for providing core/unrestricted support.

Contributors The study concept was developed by FC and PD. The protocol was drafted by PD and critically revised by FC, SB, MahmudurR, MahbuburR, ASMA, TS and MSF. Data extraction and quality assessment were performed by ZA,
SM-E-M, MAl and PD. Laboratory aspect was managed by MZR and MustafizurR. PD developed the first draft of the manuscript. MahbuburR, MMB, SB and all other authors provided feedback for all sections of the protocol including the design, analysis and revision of the manuscript. FC is the guarantor of this work.

Funding This research protocol was funded by the Foreign, Commonwealth \& Development Office (FCDO-former DFID) of the UK Government (01831) and the US Agency for International Development (USAID) under the terms of USAID's Alliance for Combating TB in Bangladesh activity cooperative agreement no. CA \# 72038820CA00002.

Disclaimer Our funding sources had no role in the design of the protocol, and were not involved during the methodological execution, data analyses and interpretation and decision to submit or to publish the study results. Views expressed herein do not necessarily reflect the views of the US Government or USAID.

Map disclaimer The depiction of boundaries on this map does not imply the expression of any opinion whatsoever on the part of BMJ (or any member of its group) concerning the legal status of any country, territory, jurisdiction or area or of its authorities. This map is provided without any warranty of any kind, either express or implied.

Competing interests None declared.

Patient consent for publication Not required.

Ethics approval The protocol was reviewed and approved by the institutional review boards (IRB; Research Review Committee and Ethical Review Committee) of the International Centre for Diarrhoeal Disease Research, Bangladesh (ref. number PR-20032). We obtained written, informed consent of the participants before enrolment. To ensure anonymity of the study participants and maintain the confidentiality, the names and identifying information of the participants were and will not be shared with anyone outside of the data collection team and this information was kept in locked cabinets and/or computers with passwords. Laboratory specimens were identified only by patient enrolment ID. Necessary permission was obtained from the respective hospitals before establishing the hospital-based platform and data collection.

Provenance and peer review Not commissioned; externally peer reviewed.

Data availability statement Data are available upon reasonable request. Data cannot be shared publicly because they are confidential. Data are available from the respective department of icddr,b (www.icddrb.org) for researchers who meet the criteria for access to confidential data.

Supplemental material This content has been supplied by the author(s). It has not been vetted by BMJ Publishing Group Limited (BMJ) and may not have been peer-reviewed. Any opinions or recommendations discussed are solely those of the author(s) and are not endorsed by BMJ. BMJ disclaims all liability and responsibility arising from any reliance placed on the content. Where the content includes any translated material, BMJ does not warrant the accuracy and reliability of the translations (including but not limited to local regulations, clinical guidelines, terminology, drug names and drug dosages), and is not responsible for any error and/or omissions arising from translation and adaptation or otherwise.

Open access This is an open access article distributed in accordance with the Creative Commons Attribution Non Commercial (CC BY-NC 4.0) license, which permits others to distribute, remix, adapt, build upon this work non-commercially, and license their derivative works on different terms, provided the original work is properly cited, appropriate credit is given, any changes made indicated, and the use is non-commercial. See: http://creativecommons.org/licenses/by-nc/4.0/.

ORCID iDs

Pritimoy Das http://orcid.org/0000-0001-8353-120X

Zubair Akhtar http://orcid.org/0000-0001-5054-9243

\section{REFERENCES}

1 Cheng ZJ, Shan J. 2019 novel coronavirus: where we are and what we know. Infection 2020;48:155-63.

2 Worldometer. Coronavirus update (live): cases and deaths from COVID-19 virus pandemic. Available: https://www.worldometers.info/ coronavirus/ [Accessed 1 Apr 2021].

3 Huang C, Wang Y, Li X, et al. Clinical features of patients infected with 2019 novel coronavirus in Wuhan, China. The Lancet 2020;395:497-506.

4 Leung $C$. Clinical features of deaths in the novel coronavirus epidemic in China. Rev Med Virol 2020;30:e2103. 
5 Hassan SA, Sheikh FN, Jamal S, et al. Coronavirus (COVID-19): a review of clinical features, diagnosis, and treatment. Cureus 2020.

6 Oran DP, Topol EJ. Prevalence of Asymptomatic SARS-CoV-2 Infection : A Narrative Review. Ann Intern Med 2020;173:362-367.

7 WHO Timeline - COVID-19.

8 COVID-19 Map - Johns Hopkins Coronavirus Resource Center. Available: https://coronavirus.jhu.edu/map.html [Accessed 25 Mar 2020].

9 The first few $X$ cases and contacts (FFX) investigation protocol for coronavirus disease 2019 (COVID-19), version 2.2. Available: https://www.who.int/publications/i/item/the-first-few-x-cases-andcontacts-(-ffx)-investigation-protocol-for-coronavirus-disease-2019(-covid-19)-version-2.2 [Accessed 15 Nov 2020].

10 Ahmed M, Aleem MA, Roguski K. Estimates of seasonal influenzaassociated mortality in Bangladesh, 2010-2012. Influenza Other Respi Viruses 2018;12:65-71.

11 Zaman RU, Alamgir ASM, Rahman M, et al. Influenza in outpatient IL case-patients in national hospital-based surveillance, Bangladesh, 2007-2008. PLoS One 2009;4:e8452.

12 Islam MT, Talukder AK, Siddiqui MN, et al. Tackling the COVID-19 pandemic: the Bangladesh perspective. J Public Health Res 2020;9:1794

13 Bangladesh Coronavirus: 430,496 Cases and 6,173 Deaths Worldometer. Available: https://www.worldometers.info/coronavirus/ country/bangladesh/ [Accessed 15 Nov 2020].

$14 \mathrm{WHO}$. Updated who recommendations for international traffic in relation to COVID-19 outbreak. Available: https://www.who.int/ news-room/articles-detail/updated-who-recommendations-forinternational-traffic-in-relation-to-covid-19-outbreak [Accessed 2 Sep 2021].

15 Mamun MA. Exploring factors in fear of COVID-19 and its GIS-based nationwide distribution: the case of Bangladesh. BJPsych Open 2021;7:e150.

16 Mah-E-Muneer S, Hassan MZ, Biswas MAAJ, et al. Use of antimicrobials among suspected COVID-19 patients at selected hospitals, Bangladesh: findings from the first wave of COVID-19 pandemic. Antibiotics 2021;10. doi:10.3390/antibiotics10060738. [Epub ahead of print: 1806 2021].

17 Covid-19 tests: technicians to be trained on PCR machines. Available: https://thefinancialexpress.com.bd/health/covid-19-teststechnicians-to-be-trained-on-pcr-machines-1585971746 [Accessed 2 Sep 2021].

18 Summ ML-MS, 2012 undefined. Global health surveillance. cdc. gov. Available: https://www.cdc.gov/mmWR/preview/mmwrhtml/ su6103a4.htm [Accessed 23 Sep 2021].

19 Ibrahim NK. Epidemiologic surveillance for controlling Covid-19 pandemic: types, challenges and implications. $J$ Infect Public Health 2020:13:1630-8.

$20 \mathrm{Ng} \mathrm{Y,} \mathrm{Li} \mathrm{Z,} \mathrm{Chua} \mathrm{YX,} \mathrm{et} \mathrm{al.} \mathrm{Evaluation} \mathrm{of} \mathrm{the} \mathrm{Effectiveness} \mathrm{of}$ Surveillance and Containment Measures for the First 100 Patients with COVID-19 in Singapore - January 2-February 29, 2020. MMWR Morb Mortal Wkly Rep 2020;69:307-11.

21 Pung R, Chiew CJ, Young BE, et al. Investigation of three clusters of COVID-19 in Singapore: implications for surveillance and response measures. Lancet 2020;395:1039-46.

22 COVID-19 Bangladesh situation reports. who Bangladesh. Available: https://cdn.who.int/media/docs/default-source/searo/bangladesh/ covid-19-who-bangladesh-situation-reports/who-covid-19-update29-20200914.pdf?sfvrsn=bd0e839f_2 [Accessed 23 Sep 2021].

23 Kushwaha S, Khanna P, Rajagopal V, et al. Biological attributes of age and gender variations in Indian COVID-19 cases: a retrospective data analysis. Clin Epidemiol Glob Health 2021;11:100788.

24 Ahmed SM, Adams AM, Chowdhury M, et al. Gender, socioeconomic development and health-seeking behaviour in Bangladesh. Soc Sci Med. 2000;51:361-71.

25 More men than women dying in BD from coronavirus. Available: https://www.thefinancialexpress.com.bd/national/more-men-thanwomen-dying-in-bd-from-coronavirus-1587215821 [Accessed 24 Nov 2020].
26 Chatterjee P, Anand T, Singh KJ, et al. Healthcare workers \& SARSCoV-2 infection in India: A case-control investigation in the time of COVID-19. Indian J Med Res 2020;151:459-67.

27 Hassan MZ, Monjur MR, Styczynski AR. Protecting front line health care workers should be the top priority in low-resource health systems: case of Bangladesh. Infect Control Hosp Epidemiol 2020;1.

28 Yasmin R, Parveen R, Azad NA, Al AN, et al. Corona virus infection among healthcare workers in a COVID dedicated tertiary care hospital in Dhaka, Bangladesh. J Bangladesh Coll Physicians Surg 2020:43-9.

29 COVID-19 Dashboard, Bangladesh. Available: http://dashboard. dghs.gov.bd/webportal/pages/covid19.php [Accessed 23 Sep 2021].

30 Bangladesh: who coronavirus disease (COVID-19) Dashboard with vaccination data / who coronavirus (COVID-19) Dashboard with vaccination data. Available: https://covid19.who.int/region/searo/ country/bd [Accessed 23 Sep 2021].

31 Huq S, Biswas RK. COVID-19 in Bangladesh: data deficiency to delayed decision. J Glob Health 2020;10:010342.

32 Manabe T, Akatsu H, Kotani K, et al. Trends in clinical features of novel coronavirus disease (COVID-19): a systematic review and meta-analysis of studies published from December 2019 to February 2020. Respir Investig 2020;58:409-18.

33 Yang W, Cao Q, Qin L, et al. Clinical characteristics and imaging manifestations of the 2019 novel coronavirus disease (COVID19):A multi-center study in Wenzhou city, Zhejiang, China. $J$ Infect 2020;80:388-93.

34 Rodriguez-Morales AJ, Cardona-Ospina JA, Gutiérrez-Ocampo $\mathrm{E}$, et al. Clinical, laboratory and imaging features of COVID-19: a systematic review and meta-analysis. Travel Med Infect Dis 2020;34:101623.

35 Cen Y, Chen X, Shen Y, et al. Risk factors for disease progression in patients with mild to moderate coronavirus disease 2019-a multicentre observational study. Clin Microbiol Infect 2020;26:1242-7.

36 Shi Q, Zhang X, Jiang F, et al. Clinical characteristics and risk factors for mortality of COVID-19 patients with diabetes in Wuhan, China: a two-center, retrospective study. Diabetes Care 2020;43:1382-91.

37 Livingston E, Bucher K. Coronavirus disease 2019 (COVID-19) in Italy. JAMA 2020;323:1335.

38 MIS, DGHS, Bangladesh. Available: https://dghs.gov.bd/index.php/ $\mathrm{bd} /$ component/content/article?layout=edit\&id $=5612$ [Accessed 2 Jan 2021].

39 Chen R, Liang W, Jiang M, et al. Risk Factors of Fatal Outcome in Hospitalized Subjects With Coronavirus Disease 2019 From a Nationwide Analysis in China. Chest 2020;158:97-105.

40 Wu Z, McGoogan JM. Characteristics of and Important Lessons From the Coronavirus Disease 2019 (COVID-19) Outbreak in China: Summary of a Report of 72314 Cases From the Chinese Center for Disease Control and Prevention. JAMA 2020;323:1239-1242.

41 Public Health England. Seasonal flu vaccine uptake in GP patients: winter 2018 to 2019 - GOV.UK. Available: https://www.gov.uk/ government/statistics/seasonal-flu-vaccine-uptake-in-gp-patientswinter-2018-to-2019 [Accessed 25 Nov 2020].

42 Huang $R$, Zhu L, Xue L, et al. Clinical findings of patients with coronavirus disease 2019 in Jiangsu province, China: A retrospective, multi-center study. PLoS Negl Trop Dis 2020;14:e0008280.

43 Wang D, Hu B, Hu C, et al. Clinical characteristics of 138 hospitalized patients with 2019 novel coronavirus-infected pneumonia in Wuhan, China. JAMA 2020;323:1061.

44 Guo FR. Smoking links to the severity of COVID-19: an update of a meta-analysis. J Med Virol 2020;92:2304-5.

45 Vardavas C, Nikitara K. COVID-19 and smoking: a systematic review of the evidence. Tob Induc Dis 2020;18.

46 Smoking and COVID-19. who. Available: https://www.who.int/newsroom/commentaries/detail/smoking-and-covid-19 [Accessed 2 Jan 2021].

47 Patanavanich R, Glantz SA. Smoking is associated with COVID-19 progression: a meta-analysis. Nicotine Tob Res 2020;22:1653-6. 